\title{
Computer-Aided Learning in Artificial Neural Networks
}

\author{
J.V. Ringwood*, G. Galvin** and J. Bruton*** \\ *Dept. of Electronic Eng. \\ ${ }^{* *}$ Ericsson Systems \\ NUI Maynooth \\ Radio House, Beech Hill \\ *** School of Electronic Eng. \\ Co. Kildare, Ireland \\ Clonskeagh, Dublin 4, Ireland \\ Dublin City University \\ Glasnevin, Dublin 9, Ireland
}

\begin{abstract}
This paper describes the development and evaluation of a Computer-Aided Learning (CAL) package for a graduate course in artificial neural networks. The package has been evaluated over a period of two academic years both as an educational supplement to a conventional lecture course and also as a completely self-sufficient remotely-taught course. The course is accessed via the World-Wide-Web (WWW). The course features Java applets for animation/demonstration purposes as well as employing the $\operatorname{MATLAB}^{T M}$ computational engine for interactive examples and assignments. In an effort to provide a classroom-like environment, an interactive discussion forum is provided, along with weekly lecture summaries from the conventional lecture course. Automatically marked question pools are available for self-assessment.
\end{abstract}

\section{Introduction}

In the current era of computer literacy and proliferation of personal computers, there exists considerable scope for the exploitation of computing resources for educational purposes. However, many other educational media also exist, and it is the judicious choice of appropriate computer-based materials which truly exploits this technology to its best advantage. Engineering disciplines, whose theory by nature can be computational intensive and whose understanding can be enhanced through graphical analysis, stand to benefit considerably from such technology. Additionally, there is also a need to provide an implementation which maximises the potential use of this medium, and not simply resort to nothing more than an electronic textbook. This is important if a cost-effective and invigorating environment is to be developed.

Some consideration must also be given to the suitability of computer-based educational material as either support material or a purely stand-alone package. For example, graduate students in the School of Electronic Engineering at Dublin City University (DCU) prefer to attend lectures, although the complete course is available on-line. However, this is due, in part at least, to the more traditional educational environments to which students have been exposed over a number of years, reflecting the low proliferation of more diverse educational techniques, which have recently become available [1]. Nevertheless, those students still make use of aspects of the package which are not so easily captured in the traditional setting, for example the use of multiple choice questions (MCQs) which are instantly marked on-line. 
Perhaps the most significant step in the application of technology in education comes from the marriage of computing and communications, which gives both extensive access and a powerful educational environment on the same platform [2]. It is this combination which is the focus of the educational module described in this paper. The subject area which the module addresses is Artificial Neural Networks (ANNs), which require considerable computation in their evaluation and use, making them an ideal candidate for a CAL package.

The educational module described in this paper lies within the MEng in Electronic Systems programme at DCU. This is essentially a one-year taught Master's programme, where students choose eight taught modules and complete a 4 -month project. In conjunction with this programme, there is an additional possibility of remote access for part-time students, who are generally in fulltime engineering employment. This remote access programme offers the programme modules via the internet and is termed Remote Access for Continuing engineering Education (RACeE).

\section{Motivation for CAL}

In addition to its obvious use in a distance-learning scenario, CAL can also be used to enrich the classroom experience, through the use of a data projector. However, is is in remote access mode that many of the unique advantages of CAL are realised. Nevertheless, some care needs to be given to the aspects of the classroom which has led to its success as the most prolific learning environment. The principal issues involved here are:

Pacing of learning material Material is presented in bite-sized chunks in a logical sequence, with an even pace appropriate to the course duration,

Gestures and movements add to the learning experience and give it a 'live show' feel [3],

Interpersonal skills of the lecturer enrich the learning experience and the interaction between classmates can be very beneficial [4],

The competitive environment of a classroom can be beneficial in motivating students to learn course material. However, we can also observe that such competition can also be destructive, with the possibility of unfavourable outcomes such as anxiety, low esteem and poor relations between students,

Supplementary material Course material is focussed on requirements for the given module, with students referred to supplementary material only as required [5].

Th main benefits of CAL, as used in a distance-learning environment, can be cited as:

- Self-paced learning, suitable for all abilities

- Graphics, animations and simulations are easily incorporated 
- Using hypertext, there can be many ways by which the same piece of information can be reached [6], with the caveat that some parts may never be accessed, and students may be left unsure as to whether they have covered all the necessary material [7]

- CAL is an ideal medium through which continuing professional development (CPD) may be accomplished, since the clientele is normally composed of mature learners with significant time restrictions

- Computer-based testing can easily be integrated with a CAL package, giving students instant feedback, which can be either directed [8] or undirected

- On a more subtle level, CAL offers relative freedom from discrimination due to physical characteristics (or racial/cultural factors), that may arise in the traditional classroom [9]. It also provides a solution for those with physical disabilities, for whom attending and navigating a university campus would prove too difficult.

As a facilitating platform for CAL, the Internet has received considerable attention over the last decade. With the first web browser introduced in 1993, an estimated 20 million web sites by the year 2000 and current connectivity of personal computers (PCs) increased by 45 million in the first quarter of 1998, it is clear that it is a great resource for CAL-based courses [10]. The important characteristics that relate to the used of an Internet-based CAL platform include:

- Accessibility is unsurpassed

- A diverse variety of media is supported

- Java allows for the development of interactive examples

- Communication between users and lecturers is facilitated via email and other conferencing tools such as mailing list and message boards

- Course material is held as a single copy and is easy to update

- There is access to a further wealth of related information via hyperlinks, put this has its own dangers viz. information overload.

\section{Artificial Neural Networks Course}

The ANN course, like the other modules in the MEng programme, is a 36 hour lecture course and additionally involves three assignments, each taking about 8 hours. Course lectures are scheduled for 12 weeks (giving a three-hour lecture block per week) followed by a study week and a week of exams. Assignments count 25\% towards the overall course mark. Further information on the course content can be found at [11]. The interested reader, wishing to learn more about artificial neural networks in a more conventional setting, is directed to [12]. 


\begin{tabular}{|l|c|c|}
\hline Course aspect & Year 1 (11) & Year 2 (12) \\
\hline Presentation quality & 4.27 & 4.18 \\
\hline Ease of access to course & 4.45 & 3.9 \\
\hline Ease of navigation through course & 4.36 & 4.27 \\
\hline Usefulness of MATLAB examples & 4.09 & 4.18 \\
\hline Usefulness of Java examples & 1.82 & 3.36 \\
\hline Usefulness of mailing list & 3.60 & 3.00 \\
\hline Usefulness of downloadable postscript notes & 4.30 & 4.54 \\
\hline Usefulness of additional links & 3.80 & 3.80 \\
\hline Usefulness of weekly summaries & 4.00 & 3.80 \\
\hline Usefulness of on-line quizzes & 3.45 & 3.36 \\
\hline
\end{tabular}

Table 1: Responses to MCQs

Placed against the merits and demerits of Internet-based CAL as documented in Sections 1 and 2 , this course provides an ideal test-bed for the evaluation of such an environment for the following reasons:

- The course forms a part of computer science and is very computationally intensive

- Considerable use can be made of graphics, diagrams, etc in the visualisation and analysis of ANN topology, parameters and variables

- The course is set at graduate level and students are generally experienced and mature learners, capable of pacing themselves

- The course has a CPD function as part of the RACeE programme offered by the School of Electronic Engineering at DCU

- A lecture-based course already exists upon which the CAL-based course can be built.

\section{Course Evaluation}

A student evaluation of the course is performed using a semi-structured questionnaire. Each course evaluation form contains ten MCQs and 4 free-format questions. The MCQs are graded $1 \rightarrow 5$, with 5 indicating full agreement. A sample size of $11+12$ was available, over two years of the programme with a mixture of respondents who used the course remotely and those who used it as supplementary material. Table 1 shows the MCQ responses, indicating that in general, students find the experience a very positive one, with particular emphasis on the presentation quality, course navigation, MATLAB examples and downloadable notes.

Although not widely completed by the students, those that completed the free format questions tended to use them to back up the feelings expressed in the MCQs. The main recommendation was 
the inclusion of more real-world applications and implementations of ANNs.

An attempt was made to utilise the capability of the package to record statistics on page and quiz usage. However, due to relatively small sample sizes, the results from correlating MCQ results with final exam. marks are rather unreliable. However, a correlation coefficient of 0.32 was obtained between final mark and MCQ usage and a slightly larger figure obtained when the average MCQ mark was weighted by the MCQ usage score. Most significant, however, was the consistently good results obtained from users of the MCQs, relative to non-users.

In general, we were very encouraged with the overall student reaction to the course, both in terms of the formal evaluation and informal comments from students over the duration of the course. It was particularly noteworthy that students who took the course exclusively through remote access scored highly (the top achiever of the $97 / 98$ course was a remote access student), with the course receiving considerable usage from students using it as supplemental material. However, superior marks could easily accrue from the fact that remote access students are better motivated. In many cases, more supplemental usage (in terms of page hits) per student was recorded than that for access on a purely remote basis.

\section{Conclusions}

Over the two years that the course has run, many useful spin-offs from the course have been identified, for example the introduction of engineering students pursuing final year projects or research theses in the neural network area, especially at times when the lecture course is not running. The CPD benefits have been documented previously. Also of major benefit is the geographical flexibility of the course, allowing access world-wide, within the reaches of the Internet. In areas where Internet access is a problem, or transmission speeds are prohibitively low, the course is easily accommodated on a CD-ROM, with recourse to the server required only for on-line MCQs and the bulletin board.

A considerable amount of time has been expended in the development of this course. Starting from the basic lecture course, the on-line course took approx. 2.5 man-years to develop, with many short-cuts taken in the recommended full development process for CAL courses. Fortunately, there has been minimal overhead in annual maintenance, with basic requirements being to archive annual statistics files, reset the bulletin board, provide new usernames/passwords and update the available past examination papers, in addition to any required modification to the basic course material.

One of our great disappointments was the poor utilisation of the Java examples. These were intended to provide a user-friendly, interactive introduction to course sections. However, many students skipped these altogether and did not even try them. On the other hand, students made considerable use of the MATLAB examples and this is significant, considering that most MATLAB examples were generated to produce the (lecture-based) course examples, with minimal overhead required to add some comments and provide the download mechanism. In contrast, the Java examples were provided specially for the course and took approx. 0.5 man-years to produce. This may add 
further spice to the MATLAB Vs. Java debate [13]! Other very profitable additions were the bulletin board, which is vital for remote-access students, and the MCQs which, although time-consuming to produce, seemed to be worth the effort.

Finally, we would encourage readers to view our package at http://www.eeng.dcu.ie/ annet.

\section{References}

[1] P.M. Lee and , W.G. Sullivan, "Developing and Implementing Interactive Multimedia in Education", IEEE Transactions on Education, Vol 39, no 3, August 1996, pp 430 - 435.

[2] P. Penfield and R.C. Larson, "Education via Advanced Technologies", IEEE Transactions on Education, Vol 39, no 3, August 1996, pp 436 - 443.

[3] University of Michigan. Distance Learning: Planning Considerations and Options [online]. Available from: http://www.oit.itd.umich.edu/reports/DistanceLearn/sect5.1-4.html [Accessed 14 July 1998]

[4] N.A. Springhall, C.C. Springhall and S.N. Oja, "Educational Psychology: A Developmental Approach", McGraw-Hill Inc., 6ed, 1994.

[5] A. Sloane, "Learning with the Web: Experience of using the World Wide Web in a learning Environment", Computers in Education, Vol 28, No. 4, 1997, pp 207-212.

[6] E. Hansen, "The role of interactive video technology in higher education: Case study and proposed framework", Educational Technology, Sept 1990, pp 13-21

[7] S.A. Mengel and J.A. Adams, "The need for Hypertext Instructional Design Methodology", IEEE Transactions on Education, Vol 39, No. 3, August 1996, pp 375 - 380.

[8] R. Gagne, W. Wager and A. Rojas, "Planning and authoring computer assisted instruction lessons", Educational Technology, 21, 1981, pp 17-26

[9] G. Kearsley, W. Lynch and D. Wizer, "The Effectiveness and Impact of Online Learning in Graduate Education", Educational Technology, November-December 1995, pp 35-42

[10] R.M. Starr, "Delivering Instruction on the World Wide Web: Overview and Basic Design Principles", Educational Technology, May-June 1997, pp 7-15.

[11] J.V. Ringwood and G. Galvin, Artificial Neural Networks [online]; Available from: http://www.eeng.dcu.ie/annet [Accessed 20 February 1999]

[12] S. Haykin Neural networks - A comprehensive foundation (2 ${ }^{\text {nd }}$ Ed.), Prentice Hall, 1999.

[13] S.G. Crutchfield and W.J. Rugh, "Interactive Learning for Signals, Systems and Control", IEEE Control Systems Magazine, August 1998, pp 88 - 91. 